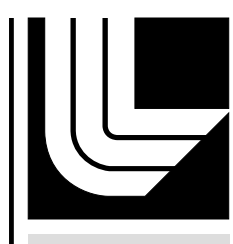

LA W REN CE LIVERMORE N A TIO NAL LABORATORY

Dynamic Behavior of Sand: Annual Report FY 11

T. Antoun, E. Herbold, S. Johnson

March 19, 2012 
This document was prepared as an account of work sponsored by an agency of the United States government. Neither the United States government nor Lawrence Livermore National Security, LLC, nor any of their employees makes any warranty, expressed or implied, or assumes any legal liability or responsibility for the accuracy, completeness, or usefulness of any information, apparatus, product, or process disclosed, or represents that its use would not infringe privately owned rights. Reference herein to any specific commercial product, process, or service by trade name, trademark, manufacturer, or otherwise does not necessarily constitute or imply its endorsement, recommendation, or favoring by the United States government or Lawrence Livermore National Security, LLC. The views and opinions of authors expressed herein do not necessarily state or reflect those of the United States government or Lawrence Livermore National Security, LLC, and shall not be used for advertising or product endorsement purposes.

This work performed under the auspices of the U.S. Department of Energy by Lawrence Livermore National Laboratory under Contract DE-AC52-07NA27344. 


\title{
Dynamic Behavior of Sand: Annual Report FY 11
}

\author{
Tarabay Antoun ${ }^{1}$, Eric Herbold, Scott Johnson ${ }^{2}$ \\ Lawrence Livermore National Laboratory \\ March 14, 2012
}

\section{Project Summary}

Currently, design of earth-penetrating munitions relies heavily on empirical relationships to estimate behavior, making it difficult to design novel munitions or address novel target situations without expensive and time-consuming full-scale testing with relevant system and target characteristics. Enhancing design through numerical studies and modeling could help reduce the extent and duration of full-scale testing if the models have enough fidelity to capture all of the relevant parameters. This can be separated into three distinct problems: that of the penetrator structural and component response, that of the target response, and that of the coupling between the two. This project focuses on enhancing understanding of the target response, specifically granular geomaterials, where the temporal and spatial multi-scale nature of the material controls its response.

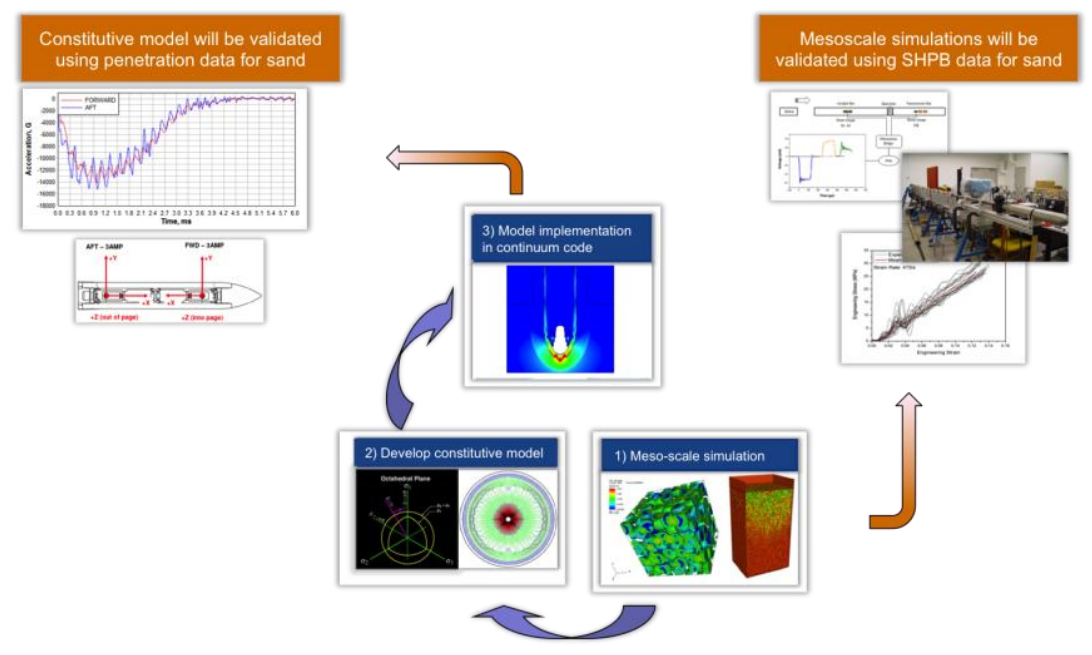

Figure 1: General approach for sequential multiscale development of enriched constitutive models for capturing behavior of sand under dynamic loading.

As part of the overarching goal of developing computational capabilities to predict the performance of conventional earth-penetrating weapons, this project focuses specifically on developing new models and numerical capabilities for modeling sand response in ALE3D.

\footnotetext{
${ }^{1}$ Principal Investigator

${ }^{2}$ Technical Lead
} 


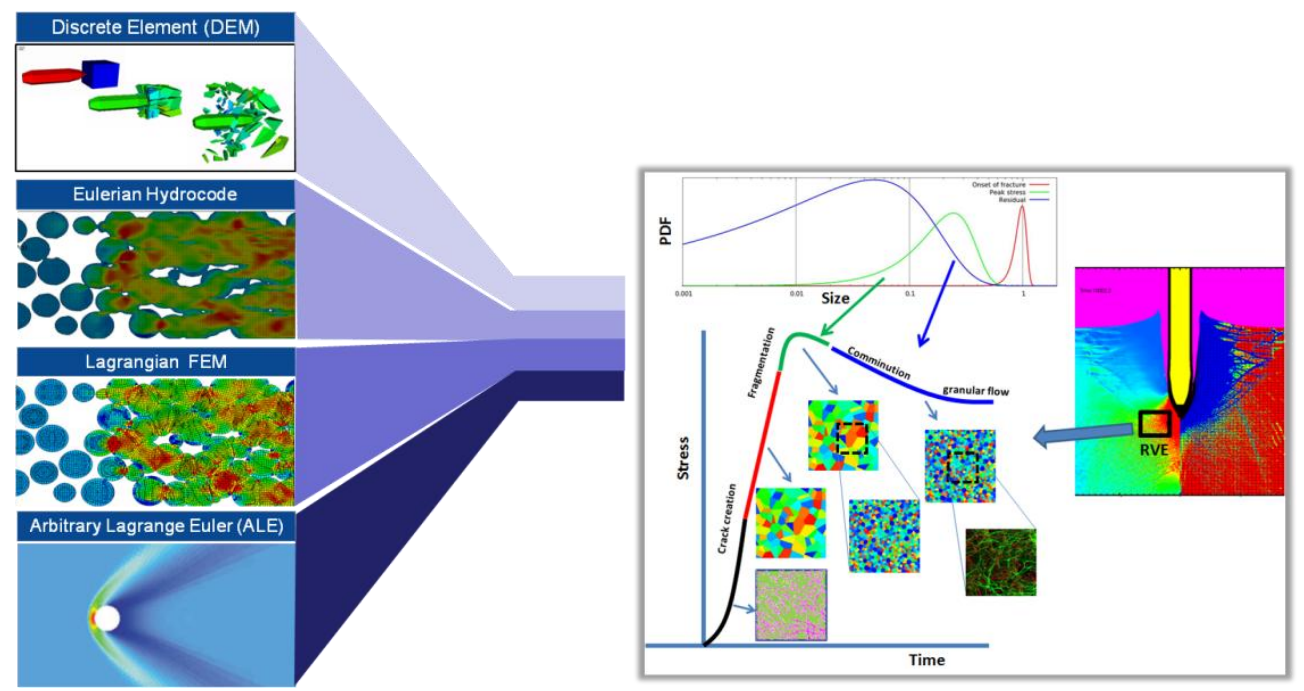

Figure 2: By combining code capabilities (left) and modeling at multiple scales (right) the entire penetration event can be captured.

There is general recognition that granular materials behave in a manner that defies conventional continuum approaches which rely on response locality and which degrade in the presence of strong response nonlinearities, localization, and phase gradients. There are many numerical tools available to address parts of the problem. However, to enhance modeling capability, this project is pursuing a bottom-up approach of building constitutive models from higher fidelity, smaller spatial scale simulations (rather than from macro-scale observations of physical behavior as is traditionally employed [1], [2]) that are being augmented to address the unique challenges of mesoscale modeling of dynamically loaded granular materials.

Through understanding response and sensitivity at the grain-scale, it is expected that better reduced order representations of response can be formulated at the continuum scale as illustrated in Figure 1 and Figure 2. The final result of this project is to implement such reduced order models in the ALE3D material library for general use.

\section{Work Prior to FY11}

Our first task was to characterize sand response from a historical, experimental, and numerical perspective. Of primary importance in this task has been to identify, isolate, and analyze different properties characterizing sands to understand the sensitivity of the overall response to the properties. To date, the literature review has allowed us to remove properties from consideration and focus on more relevant characteristics. The set of parameters that we identified for further consideration includes: sample size dependence, material density, bulk density, inter-granular friction, sizedistribution, granular fabric tensor, comminution and fracture toughness, deformability, and saturation.

For instance, intact material density and inertial terms in general have little effect on behavior in quasistatic loading conditions where stress equilibrium is achieved, while inter-granular friction appears to have a significant effect on bulk material response due to its contribution to shear strength and stability 
at the grain-scale. While overall bulk density contributes significantly to the ballistic efficiency of a particular type of sand, parameterizing this property as a function of size distribution and porosity (which encompasses effects from bulk density and from material compaction) yields a better response characterization.

\section{Parametric Studies}

A significant focus of the first part of this study is to understand the sensitivity of the bulk material behavior to various measurable grain-scale properties. The original approach envisioned for calibration and matching available, low-velocity experimental data (polyhedral discrete elements with fracture) is appropriate for impacts at less than a relative velocity of $10 \mathrm{~m} / \mathrm{s}$ and sufficiently elastic-brittle materials. Sand has a relatively high Hugoniot elastic limit of around $5 \mathrm{GPa}$ [3] and a much lower tensile strength, suggesting that bulk material damage is dominated by comminution rather than plastic deformation of the intact material. Several studies have pointed towards this being the case both from single grain experiments [4], sand box penetration experiments [5-7], and high rate loading experiments [8], [9]. However, the assumption of stress equilibrium, which places limits on the relative impact speed [10], is implicit in the discrete element (DEM) formulation, which is deleterious to extending the analysis methodology to higher velocity impacts. Therefore, the protocol has been to apply the DEM analysis to all appropriate experiments while also providing analogous full finite element solutions for a subset of the studies, thereby both confirming our initial assumptions and providing a measure of cross-code validation for studies using the full finite element solution at higher rates.

\section{Representative Volume Element}

Sample geometry and size-to-particle ratio can be important in terms of boundary effects. As a generalization a 2:1 aspect ratio of the test sample along with at least 20 grains across the least dimension were found to be sufficient to provide convergence and is as expected from experimental work in the literature.
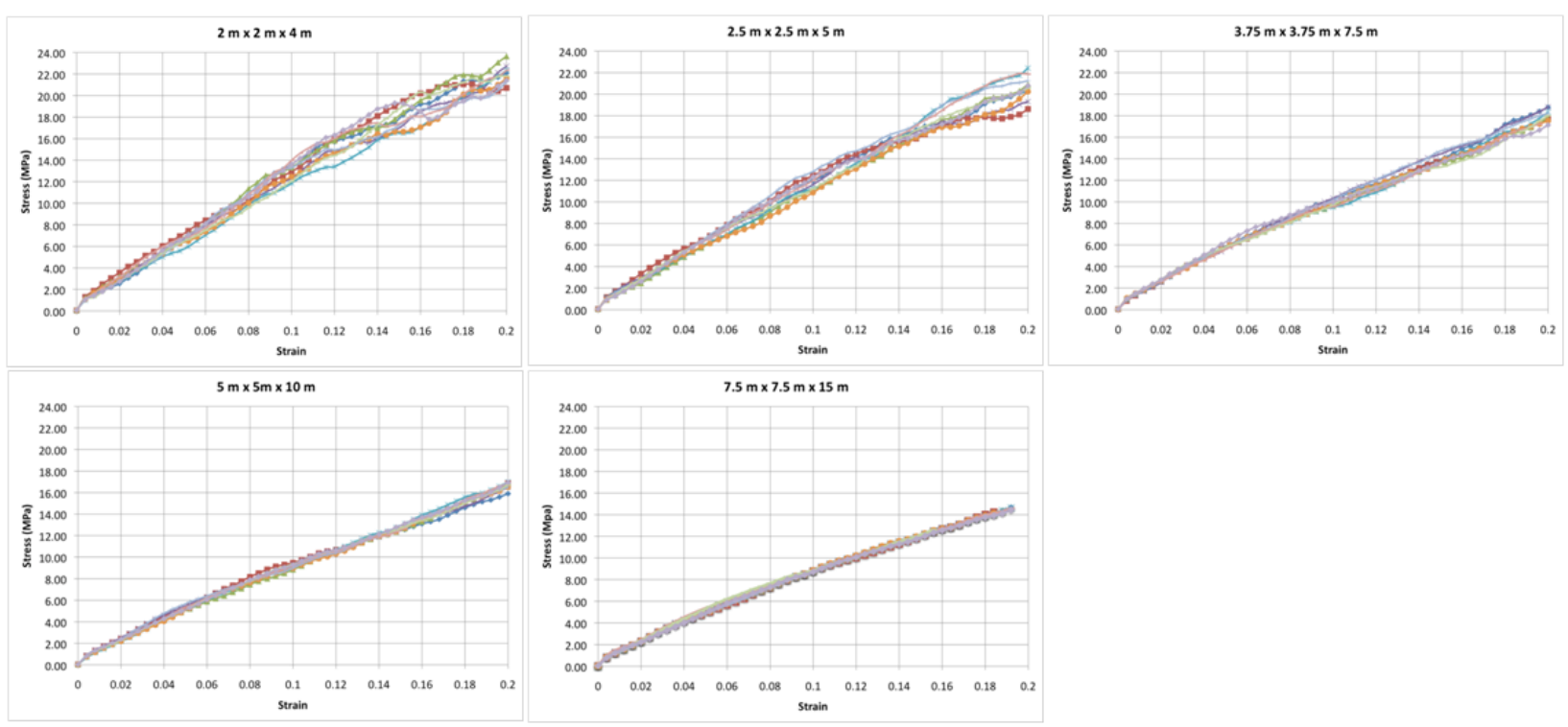
Figure 3: Stress-strain relationship for discrete element simulations varying the grain diameter to sample diameter ratio.
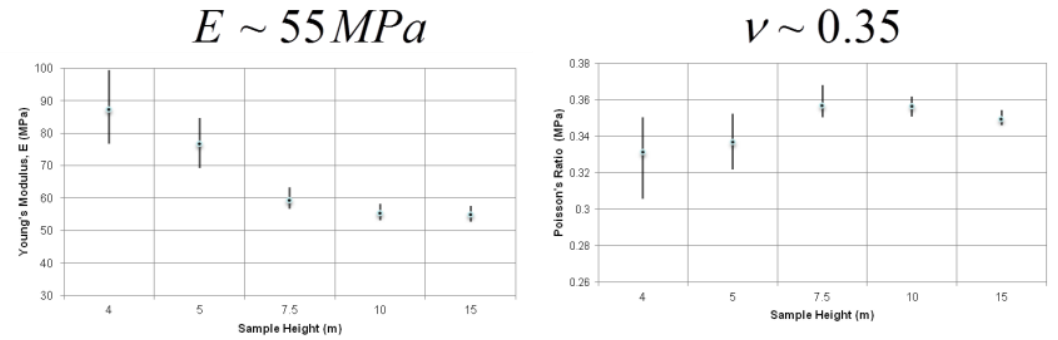

Figure 4: Bulk modulus and Poisson's ratio and range at $10 \%$ strain for a series of stochastic realizations of the granular system.

In general, the particle diameter to sample diameter ratio where the results converge is similar for both discrete element and finite element solutions as expected. Figure 5 shows that the relationships between stress and strain are quite similar between samples with 14 and 20 particles across (see curves $A$ and $B$ in Figure 5). These finite element simulations consisted of up to 17,186 spherical particle meshes (>500,000 elements) deformed uniaxially along their longest dimension, respectively. The deviations from a smooth curve in $A, B$ and $C$ are due to particle deformation and rearrangement since particle fracture was not permitted in these simulations. The severity of these deviations is affected by inter-particle friction which was also not included in these simulations. For example, friction between particle meshes, exacerbated by increasing coordination number, may prohibit particle rearrangement and particles would experience much more deformation when trapped between other particles.
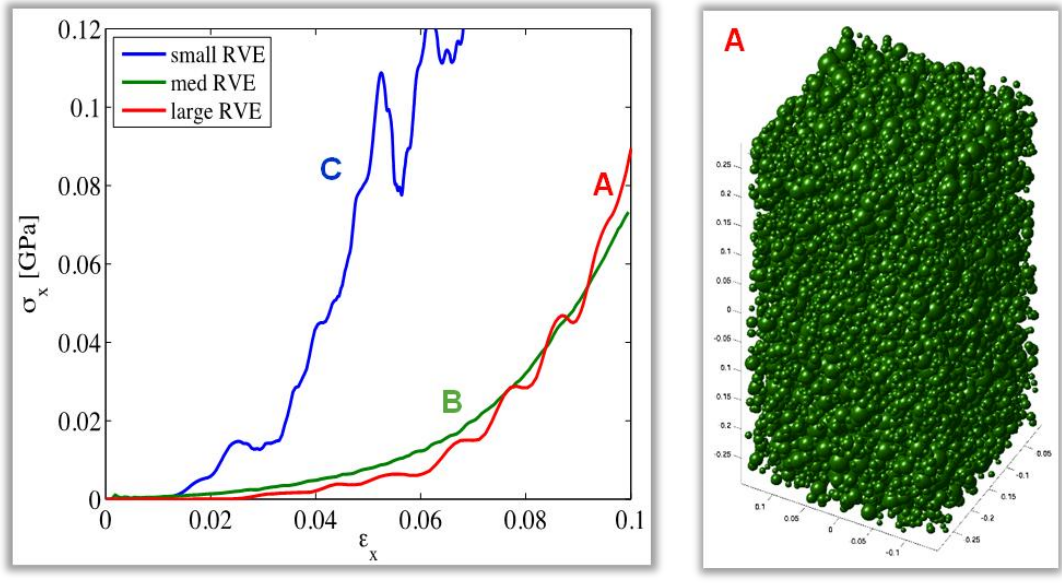

17186 particles ( 20 particles across)

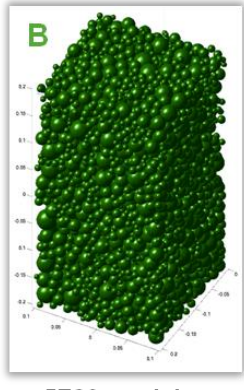

5729 particles ( 14 across)

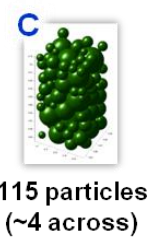

Figure 5: Effect of sample size on effective bulk modulus under uniaxial compression for deformable finite elements

\section{Size Distribution of Sample}

The size distribution of the grains also has a pronounced effect on the response of sand in general. As size distribution approaches mono-dispersity in the absence of grain crushing, both the effective bulk and shear moduli of the material increase as shown in Figure 6. These results are supported by experimental observations of decreasing shear modulus with increasing gradation [11]. On the right side of Figure 6, three simulations are shown where ceramic grains are initially tightly packed within a 
rectangular domain. Samples $A, B$, and $C$ each have different size distributions and strength response shown in their respective curves. The similarities between these simulations (e.g. similar to compression of intact polycrystalline ceramic materials) and porous sand compaction are observed by comparing the curves shown in the left and right sides of Figure 6.
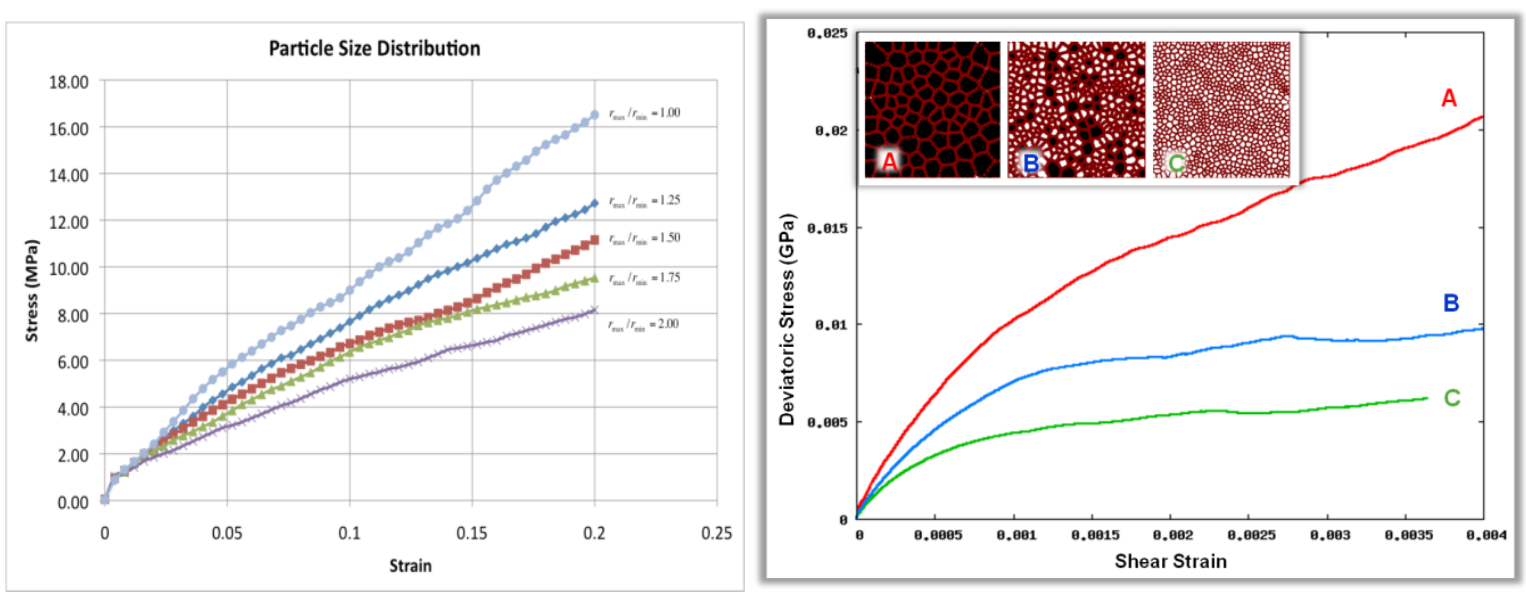

Figure 6: Effect of size distribution on bulk modulus (left) and shear modulus (right) for discrete and finite elements, respectively.

\section{Fracture}

One of the little understood parameters in granular material behavior is the effect of grain crushing (comminution) on the mechanical response. This is a difficult physical process to model with accurate fidelity. In order to simulate a statistically representative number of granular systems, a lower fidelity model of the grain crushing process is necessary. However, it is desirable to verify the model with either experiment or high fidelity modeling. In this case, both sources were sought, and the latter approach has the additional benefit of providing a measure of verification to the hybrid finite-discrete element type methodology to be used in characterizing higher rate material response.

First, we will describe the size dependent strength criterion used by the polyhedral discrete element approach. From Figure 7, based on the results of Gallagher [4], it can be seen that the trend and statistics appear to be readily characterized by a stochastic, size-dependent strength criterion. For the DEM-based part of this study, the following functional form has been adopted:

$$
Y(\phi)=\left\{\begin{array}{cc}
\left(\left(\frac{\phi_{c}-\phi_{\infty}}{\phi-\phi_{\infty}}\right)^{\alpha}(1-\gamma)+\gamma\right. \\
{[X] Y_{\text {sio } 2}} & , \quad \text { else }
\end{array}\right.
$$




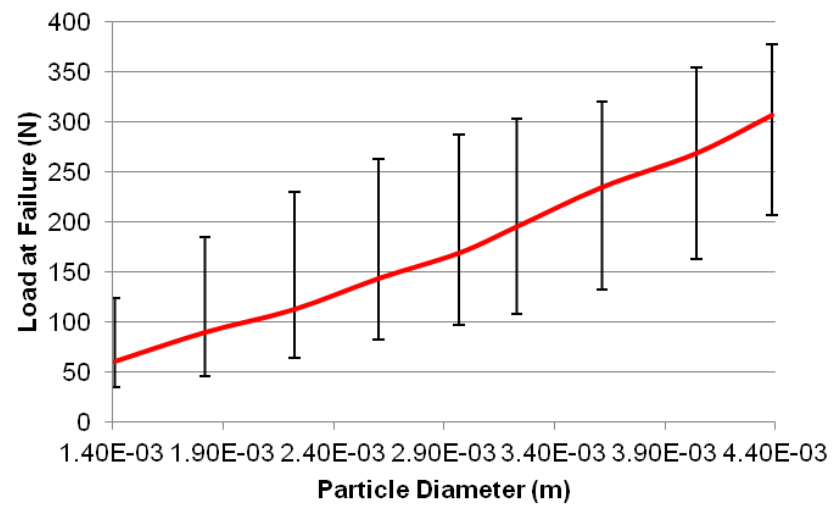

Figure 7: Range and mean of compressive load at failure for tests of individual sand grain strength

A reasonable model of fracture is achieved with arbitrary polyhedral discrete elements informed by a homogenous stress field inferred from the applied boundary forces. This is constructed as described by Morris and Johnson [12]. The result is a mechanism to cause instantaneous fracture perpendicular to the directions of least compressive stress with estimates of spatially distributed stress intensity factor (SIF) associated with the spatial distribution of load application. The resultant model yields a rich array of qualitatively and quantitatively verifiable behaviors. This is demonstrated by the comparison of pairwise brittle sphere impact for experiment [13] in Figure 8 and simulation as well as the system level damage characteristics observed during high strain, quasi-static experiments [14] and that seen in a cross-section of a simulated high-strain, quasi-static uniaxial compression experiment in Figure 9.

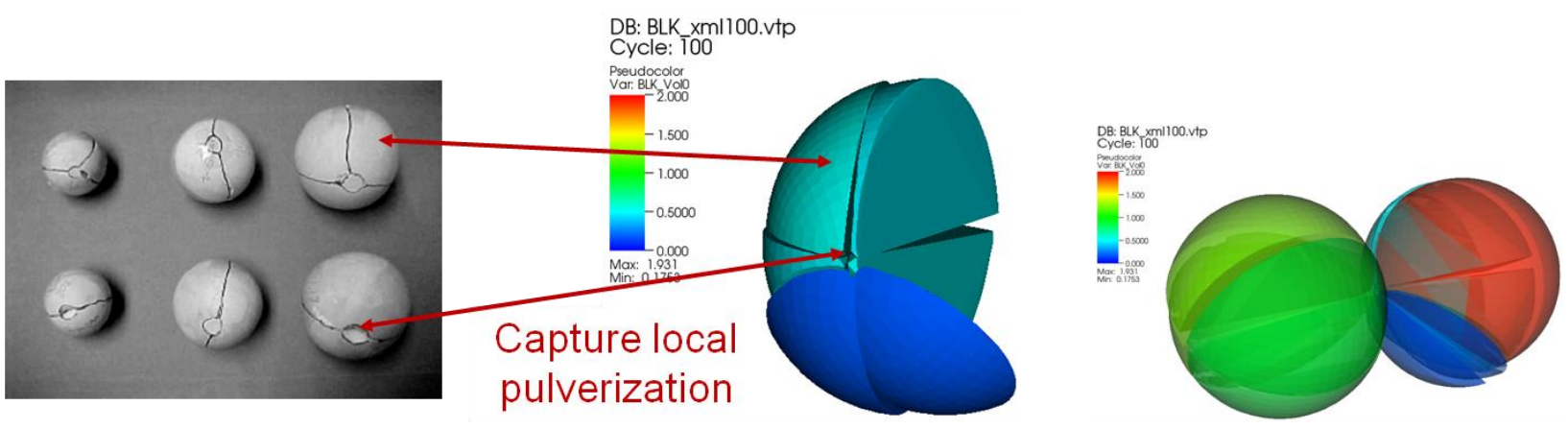

Figure 8: Qualitative comparison of resultant comminuted material from the impact of two elastic-brittle spheres (left) experiment and (right) simulation using stochastic, size-dependent strength model. 

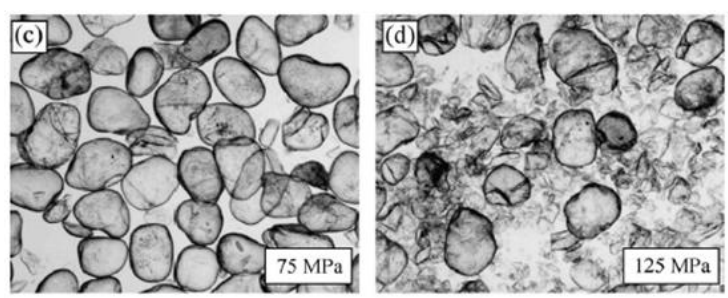

DB: BLK $x$ ml097.vtp
Cycle: 97

Pseudocolor

Var: BLK 0.02000

$-0.01500$

$-0.01000$

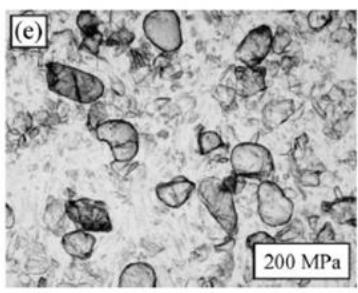

$-0.005000$

$-0.000$

Min: $4.179 \mathrm{e}-06$

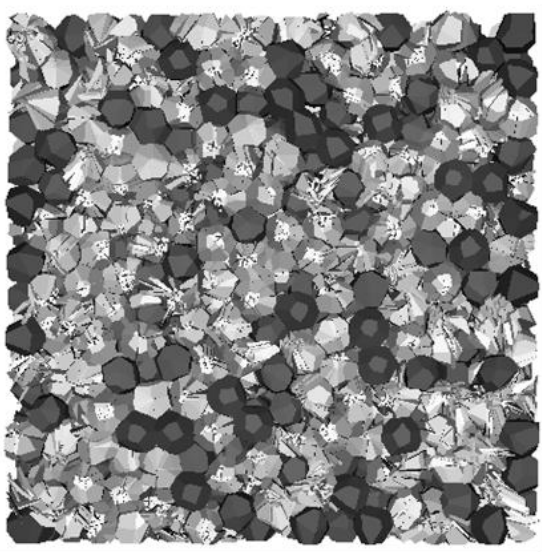

Figure 9: Distribution and extent of damage in simulation compares favorably with observations.

Preliminary results compared with a simulation of a similar system using deformable elements also show agreement (see Figure 10 vs Figure 12). Using the same code with sub-discretization of the polyhedral elements into Cosserat finite elements with spatially heterogeneous (via a fractal distribution) tensile strength also suggests that the lower fidelity model reproduces similar topology and strength as a more resolved simulation as illustrated in the representative fracture event depicted in Figure 12. Finally, the system level (mesoscale) behavior of a uniaxially loaded cell displays similar alternating strain-softening and hardening as seen in experiments, as illustrated in Figure 11 for the case of finite and infinite tensile strength grains.
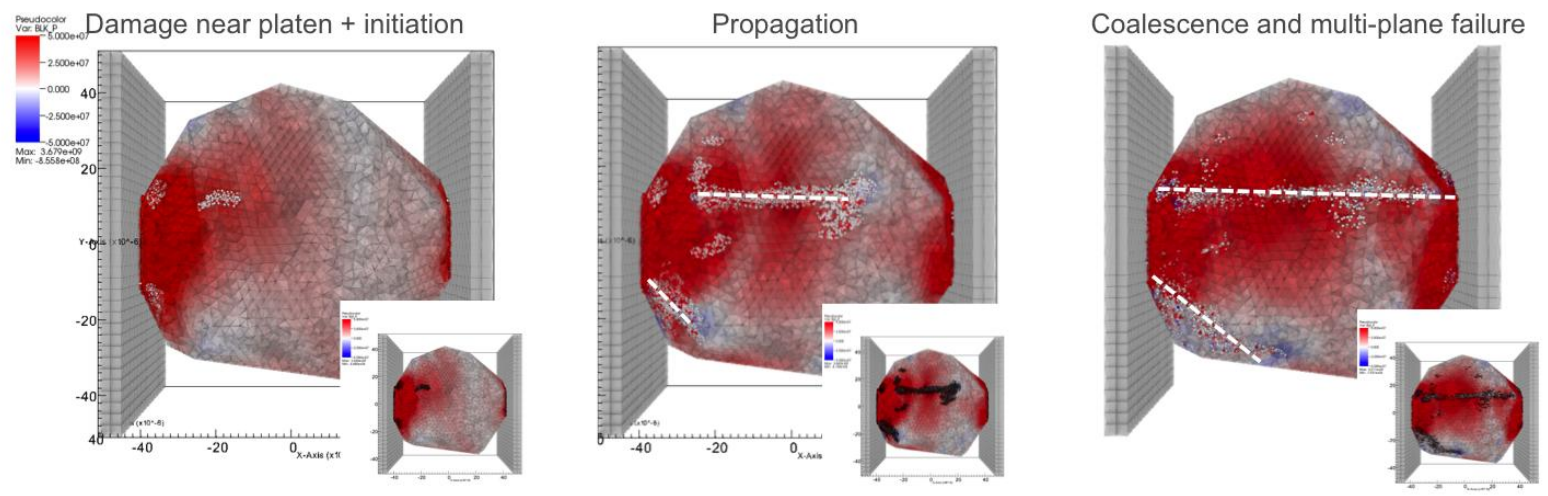

Figure 10: Higher-fidelity Cosserat finite element simulation of single grain fracturing. 

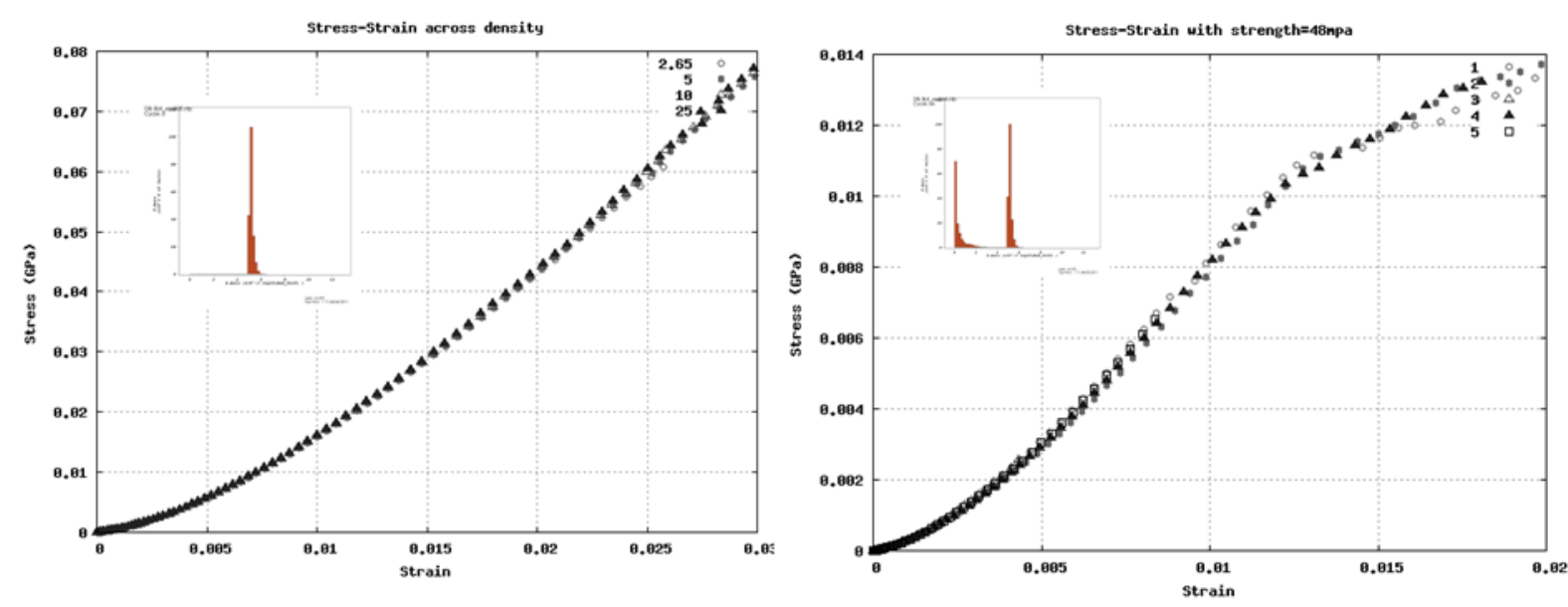

Figure 11: Effect of friability on both the evolution of the size distribution as well as the bulk modulus of the material (left) infinite strength (right) calibrated stochastic, size-dependent strength model.

Limited comparison with experiment [15] generally agree for the small strain case, but more work is necessary to conclusively establish validation.
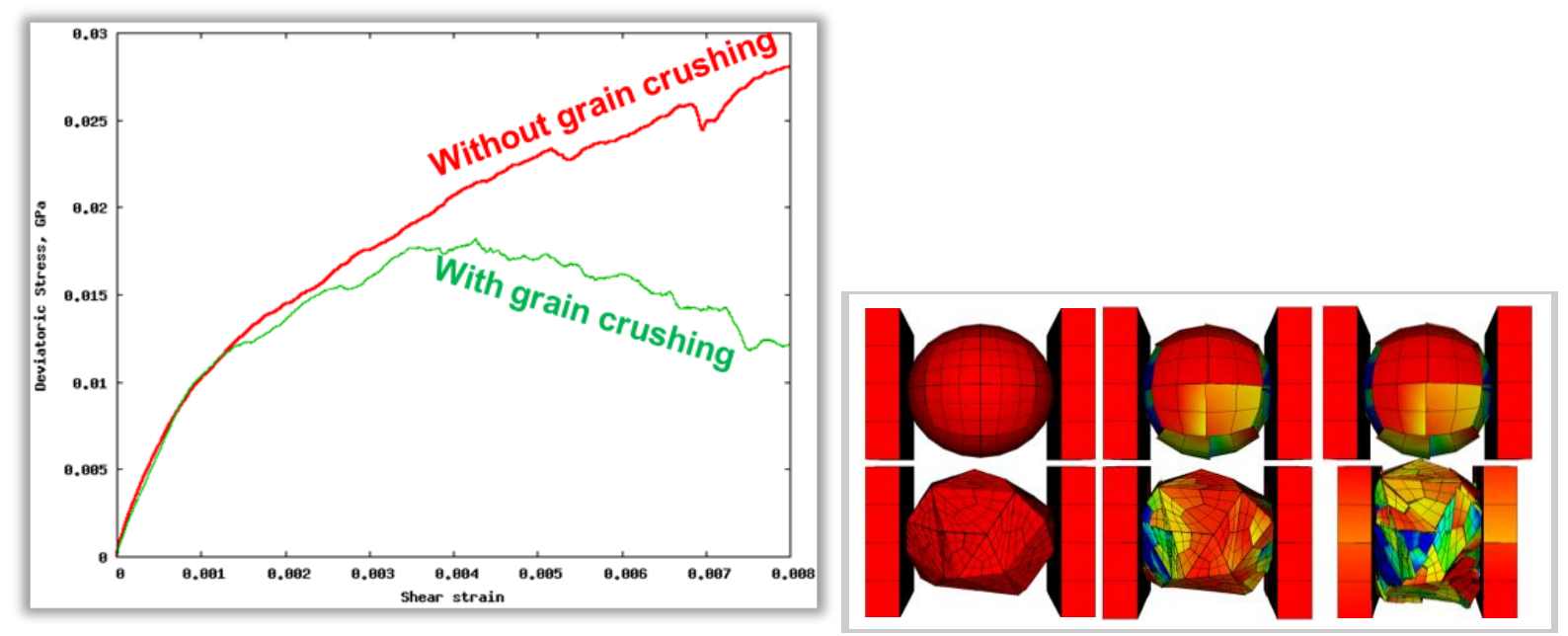

Figure 12: Effect of comminution on material response (left) characteristic single-grain tests representative of extrema of angularity range for a higher order finite element representation.

In addition to modeling the deformation of sand grains with Cosserat deformable discrete elements, finite element simulations must be performed to validate the DEM simulations. This task is complicated by the meshing technique utilized to create the grain packing (described in the sections below). Fracture of the mesh is accommodated by a cohesive contact where element faces are held together against tensile and shear motion until a sufficient amount of damage is accumulated. In Figure 13 the particle packing shown for sample B in Figure 5 is used to compose an RVE surrounded by steel walls and platens. Each platen is directed towards the center of the sample providing a uniaxial loading of the sample that includes both deformation and fracture. A cut-away image is shown in the center of Figure 13 where non-persistent 'force-chains' are clearly visible. These force chains are illustrated by values of von Mises stress in the figure and indicate that these particular particles are bearing the majority of the 
load. It is interesting that there are a large number of particles experiencing very little stress throughout the deformation. The fractured particles are shown in an expanded view on the right of the image. Note that the fracture lines are predetermined by the cohesive faces initialized in the simulation.
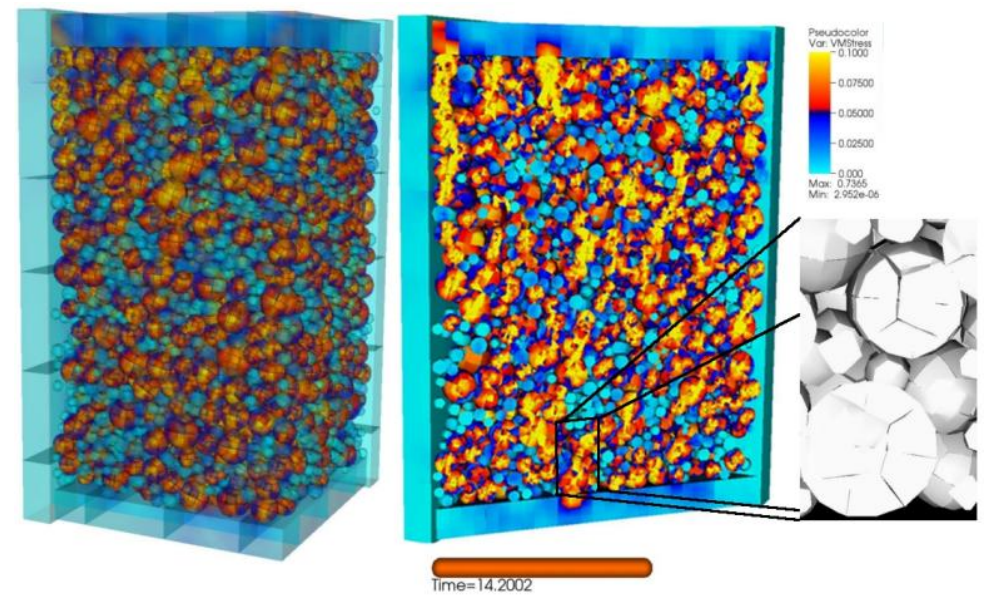

Figure 13: Finite element simulation of $\mathbf{5 7 2 9}$ deformable sand grains including fracture across cohesive faces. The calculation consisted of over 200,000 elements and was run for $>40,000$ cycles on 96 CPU's ( 20hrs). Force chains are clearly visible in the center part of the figure. The right portion of the figure shows the fractured grains.

Figure 14 shows the stress and strain response from four different simulations similar to that shown in Figure 13. In parts (a), (b) and (c) the friction coefficient between particles was equal to zero. Curve (b) shows the result for particles that are deformable, but fracture was not included in the simulation. Curve (a) corresponds to the case when grain fracture occurs at $50 \mathrm{MPa}$ in tension. Note that the deviation of this curve from the case without fracture (curve (b)) is visible after the sample has underwent $10 \%$ strain. It is interesting that this deviation occurs at around $12 \mathrm{MPa}$ here which is also the case shown in Figure 11 for fracture of a single discrete element. However, in Figure 11 the strain at fracture is much less than for the finite element RVE shown in Figure 13. This may be due to particle rearrangement during compaction as well as deformation of the finite elements. Curve (c) shows the case where the fracture strength is negligible. This highlights the effects of particle fracture strength in comparison with curve (a). Curve (d) is similar to curve(a) except that the friction between grains is much higher (friction angle $=21^{\circ}$ ). It is clear that friction between particles plays a significant role in the stress-strain response regardless of particle fracture. 


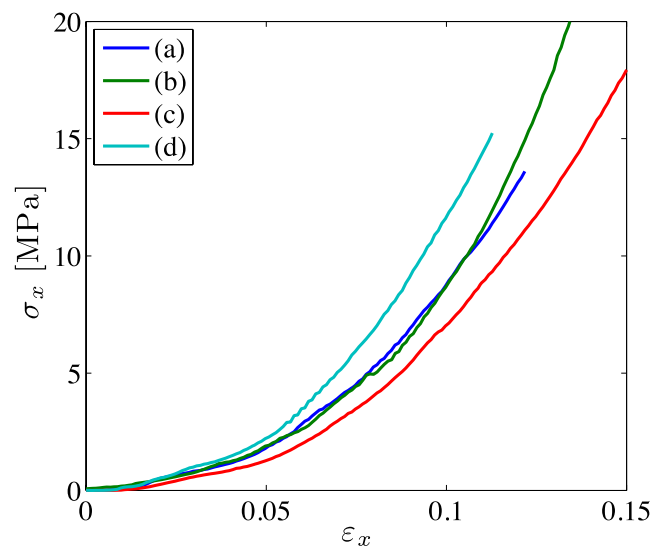

Figure 14: Stress and strain curves for four different simulations similar to that shown in Figure 13. (a) Includes fracture and a low friction coefficient between grains. (b) No fracture with low friction between grains. (c) Includes very weakly bonded grains and low fracture. (d) Includes fracture and high friction between grains.

\section{Effect of Morphology}

The fabric of the granular material can also be important in multiple ways, as has been illustrated in other studies [16] and as shown for porous and weakly consolidated materials (see Figure 15). This will be discussed for unconsolidated sands after discussing complexities in realizing systems that can probe the effects of granular fabric. That is, care must be taken to construct physically realistic packing of granular materials for different porosities and confinement.

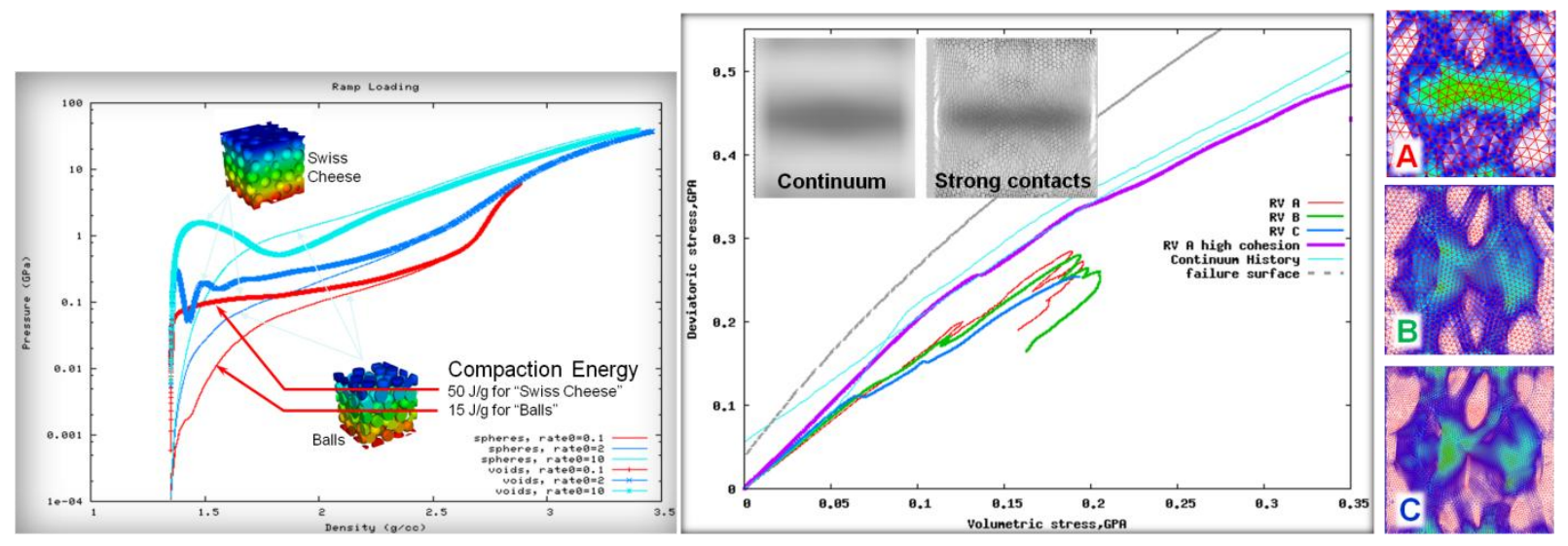

Figure 15: Effect of fabric on pressure-density response (left) and effect of interfacial cohesiveness with Weibull distribution of strength (right)

\section{Development of Packing Algorithms}

One of the issues with performing mesoscale simulations is the paucity of available algorithms to generate large systems of contacting grains. Unfortunately, the arbitrary polyhedral elements used in the lower fidelity discrete element method calculations require a different packing algorithm than that for hexahedral element meshed sand grains or even those using tetrahedral elements. To enable cross- 
code verification, it is necessary to develop appropriate model generation algorithms that optimally leverage a common algorithmic basis.

Here, all of the approaches presented share a common pre-conditioning step, where randomly placed spherical kernels are allowed to expand to a prescribed size (i.e., the sizes are taken from a prescribed grain size distribution from observations of Eglin sand [17]) and simultaneously are displaced to approach a minimum energy potential. This general preconditioning approach is both computationally efficient and scalable and is shown in Figure 16.
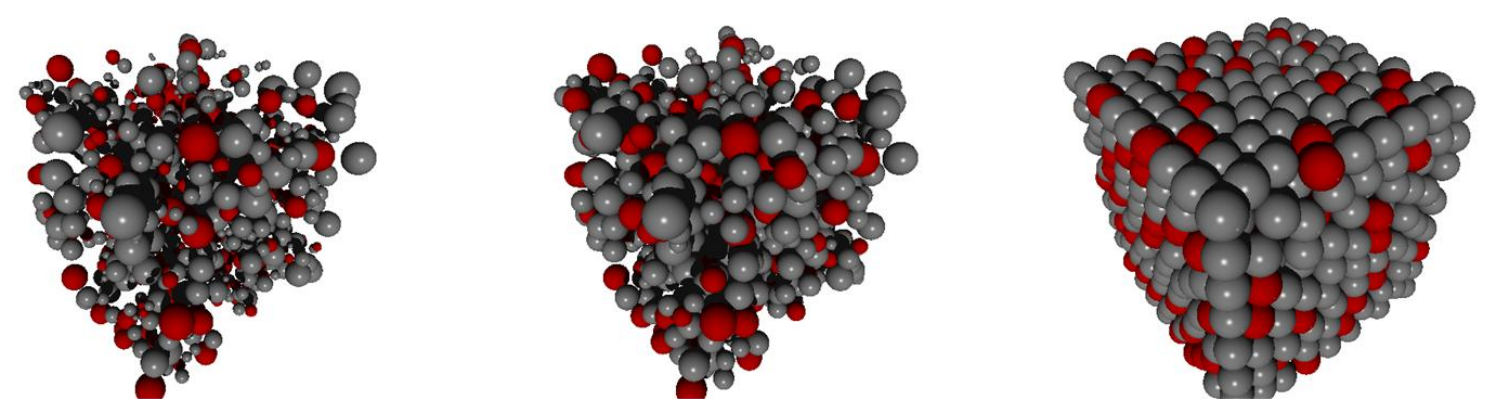

Figure 16: Illustration of placing spherical kernels randomly in a domain and then allowing the kernels to expand to a prescribed size distribution (illustrated is mono-disperse).

\section{DEM}

The discrete element variant for producing packed arbitrarily-shaped polyhedral uses the above preconditioner to provide centers of a Delaunay decomposition of the spatial domain (zero porosity). Porosity is specified by the user and an algorithm is used to order points on the surface of the polyhedron according to their divergence from the desired analytical potential function (in the illustration, the potential function is a simple scaled spherical potential), and the polyhedra are clipped via a plane perpendicular to the potential function at each point in sequence at a position defined by the point projected onto the potential surface. This destructive geometry approach is applied to each point in sequence until the desired porosity is met. The approach is illustrated in Figure 17.
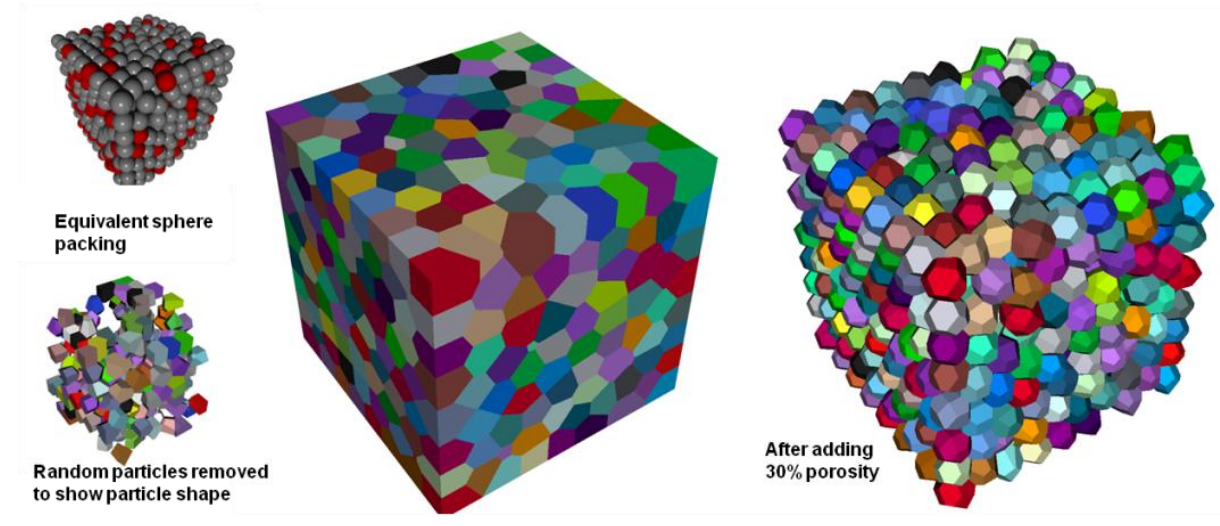

Figure 17: Illustration of packing algorithm for arbitrary polyhedra that matches size distribution and porosity simultaneously. 


\section{Tetrahedral Element Meshing}

The tetrahedral meshing of the polyhedral grains is mostly an extension of that for the polyhedral packing of the discrete elements. Each grain is treated separately, and the focus is on ensuring element quality by first templating the grain with a predefined mesh, removing elements that fall outside of the convex hull of the sand grain, reconstructing elements at the surface, then moving nodes interior to the convex hull to further optimize element quality. A manuscript detailing this approach is currently in final review for the International Journal of Numerical Methods in Engineering (IJNME).

\section{Hexahedral Element Meshing}

The extension to hexahedral meshes of arbitrary polyhedra is more nuanced. There are many cases where element quality can suffer if strict geometric compliance is required. To balance geometric fidelity with element quality and relatively equivalent element sizes, a series of spherical equi-potential meshes are constructed and cached for the range of sand grain sizes to be constructed. The resultant meshes are selected according to size, fit into the convex hull of the sand grain, then stretched to conform to the convex hull. The procedure is illustrated in Figure 19. First, a Monte-Carlo algorithm similar to [18] is implemented to pack circles (2D) or spheres (3D) of a given distribution into a spatial domain. For example, the cumulative percentage of the sand grains that pass through a certain sieve size is shown for silica based fine-grained sand in Figure 18. The sand is kiln dried and poorly graded (ASTM 2001). The black line with the circles are data points from [17]. The blue line is the result from a log-normal distribution of particle sizes where the mean particle size was specified as $126 \mu \mathrm{m}$ with a variance of 0.7 . The total number of particles sampled for the statistically generated line is 17,186 . In Figure $18(b)$ the blue line is complementary to the blue in part (a), but here the normalized particle size distribution is shown. The red line shows the result (from a different random distribution) when the particle size is limited to no less than half the size of the mean particle size and no greater than 4 times the mean. This effectively cuts the lower end of the particle size distribution without changing the specified variation. 
The result synthesizes packing with a size distribution approximating that measured from experiment and provides a spatial distribution of points from which a Lagrangian mesh can be formed. A mesh consisting of quadrilateral (2D) or hexahedral (3D) elements is then constructed about these points by using unit sized mesh templates that are transformed to fill the effective Voronoi cell about the points. This process is shown in Figure 19 where a coarse template is placed within the circular support region about one of the generated points. The black lines in the figure show the boundaries of the effective Voronoi cell about the point. A radial projection of each node is then performed to fill the void space between particles, which allows the porosity of the final packing to be arbitrarily adjusted. The result of the radial projection procedure is shown in green in Figure 19, where the limitations of the approach can be seen, i.e., a zero porosity condition cannot be strictly achieved. The initial void space is determined by the resolution of the mesh template.
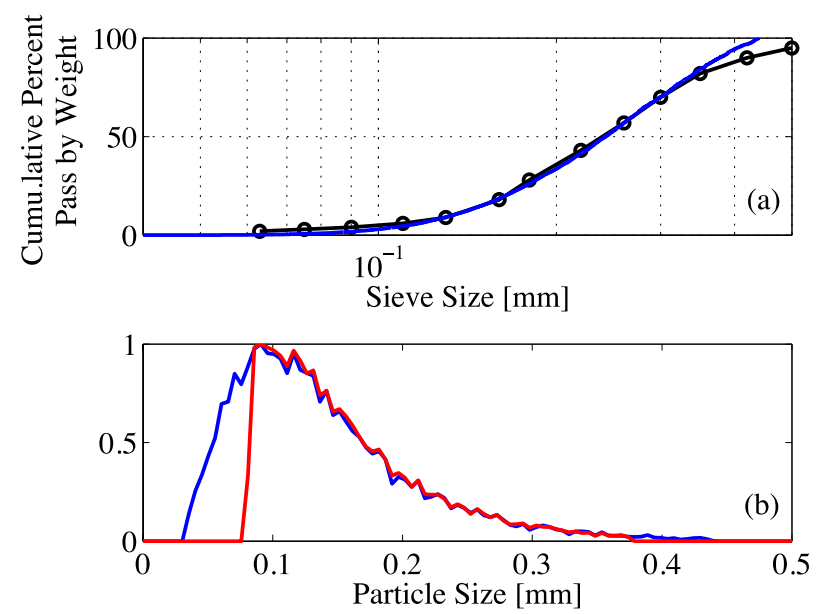

Figure 18: (a) Sieve size is shown for silica based fine grained sand. The sand ASTM 2001. (b) The blue line is complementary to the blue in in part (a). The red line shows the result cutting the lower end of the particle size distribution without changing the specified variation.

Since it is not known a priori how the distribution will change when the space is filled using the method above (Figure 19), an iterative procedure is required to determine if the desired particle size distribution is achieved. The top portion of Figure 19 shows an example where 1630 circles are packed in a rectangular domain with a normal-distributed size distribution shown by empty bins with the corresponding probability density function (PDF) as the enveloping black line $\left(R_{\text {mean }}=0.125 \mu \mathrm{m}, \sigma^{2}=\right.$ $0.01 \mu \mathrm{m}$ ). Once the mesh templates (shown on the bottom right of Figure 19) are placed and stretched to their Voronoi cell boundaries, the resulting size distribution is shown by the green bins and the enveloping curve shown is also a normal distribution PDF with $R_{\text {mean }}=0.142 \mu \mathrm{m}, \sigma^{2}=0.006 \mu \mathrm{m}$. On the bottom of Figure 19 the result of packing 7089 spheres that are replaced by spherical mesh templates, and radially stretched to their Voronoi cell boundaries. The initial normal-distributed size distribution is the same as the top part of the figure with $R_{\text {mean }}=0.125 \mu \mathrm{m}, \sigma^{2}=0.01 \mu \mathrm{m}$, but after the volume filling procedure, the bounding normal-distributed PDF is $R_{\text {mean }}=0.157 \mu \mathrm{m}, \sigma^{2}=0.24 \mu \mathrm{m}$. It is interesting that the variance decreases (from $0.01 \mu \mathrm{m}$ to $0.006 \mu \mathrm{m}$ ) in the two-dimensional case, but increases (from 
$0.01 \mu \mathrm{m}$ to $0.24 \mu \mathrm{m}$ ) in three-dimensions. Additionally, the volume-filled size distribution shown in the three-dimensional case may also be fit reasonably well with a log-normal PDF.

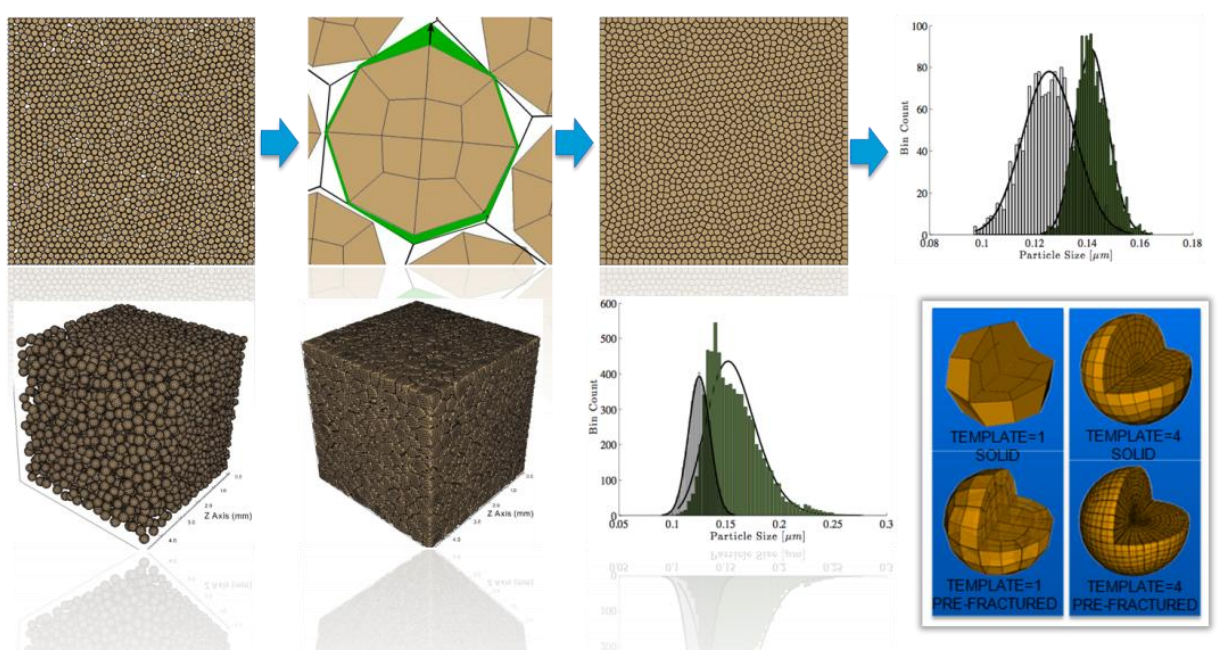

Figure 19: Illustration of the packing of hexahedrally meshed granular material.

\section{Saturation}

\section{GEODYN Simulations of Dry and Saturated Sand}

The response of saturated geologic materials is often simulated using an effective stress model, which derives the saturated material response from the dry response simply by substituting effective pressure for total applied pressure. This assumption has been validated extensively in geotechnical soil engineering applications where the strain rates and applied pressures are much lower than those encountered in dynamic applications. Experimental data for the dynamic response of saturated geologic materials in general, and sand in particular, are rare and inconclusive with regard to the applicability of the effective stress modeling assumptions. This study was undertaken to assess the effect of saturation on the response of sand under dynamic loading. Specifically, we aim to determine whether or not the effective stress model provides an adequate framework for simulating the effects of saturation on the dynamic response of sand.

Our approach relies on mesoscale simulations that resolve the details of granular mechanics during dynamic loading of dry and fully saturated sand, as shown in Figure 20. The simulations are performed using GEODYN, a massively parallel 3D Eulerian code with adaptive mesh refinement capability, and an advanced constitutive modeling framework well-suited for the response of geologic materials. 

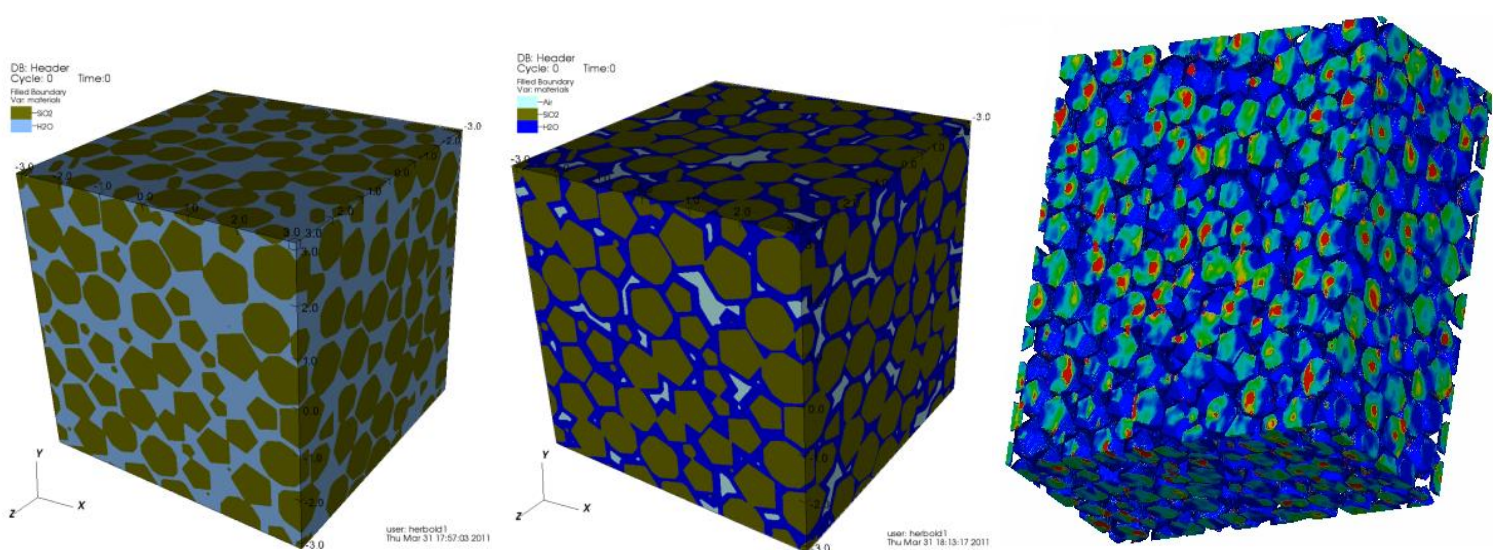

Figure 20: A snapshot showing the representative volume element (RVE) used to investigate the effect of saturation on the dynamic response of sand.

In the simulations, grains are represented as icosahedra with a nominal $1 \mathrm{~mm}$ diameter. The grains themselves are non-porous and they occupy $64 \%$ of the total volume of the representative volume element (RVE). The remaining $36 \%$ of the volume represents interstitial space, which is filled with air in the dry simulation, or water in the fully saturated simulations. The computational domain, or RVE, is a cube containing 20,000 particles. The domain size is $928 \times 928 \times 928$ computational cells, which equates to about 38 linear cells per particle diameter.

The constitutive model for $\mathrm{SiO}_{2}$, which was used to represent the response of the solid particles, employed a pressure hardening yield surface for the deviatoric behavior (shown in Figure 3), and a MieGrüneisen equation of the state with a tensile cut-off for the volumetric behavior. The elastic and EOS parameters for sand are shown in the table below.

\begin{tabular}{|l|l|}
\hline \multicolumn{2}{|c|}{ Model parameters for sand } \\
\hline Density & $2.65 \mathrm{~g} / \mathrm{cm}^{3}$ \\
\hline Sound Speed & $3.77 \mathrm{~km} / \mathrm{s}$ \\
\hline Poisson's ratio & 0.17 \\
\hline Mie-Grüneisen parameters \\
\hline $\mathrm{S}_{1}$ & 1.75 \\
\hline $\mathrm{S}_{2}$ & 0 \\
\hline $\mathrm{S}_{3}$ & 0 \\
\hline$\Gamma$ & 0.65 \\
\hline Tensile failure & $0.16 \mathrm{GPa}$ \\
\hline Pressure cut-off & 0.014 \\
\hline Volumetric Strain cut-off &
\end{tabular}

In the simulations of dry sand, air is modeled as an ideal gas with a gas constant $\gamma=1.4$. In the fully saturated simulations, water is modeled with a Mie-Grüneisen equation of state with the parameters listed in the table below.

\begin{tabular}{|l|l|}
\hline \multicolumn{2}{|c|}{ Equation of state parameters for water } \\
\hline Density & $1 \mathrm{~g} / \mathrm{cm}^{3}$ \\
\hline Sound Speed & $1.485 \mathrm{~km} / \mathrm{s}$ \\
\hline
\end{tabular}




\begin{tabular}{|l|l|}
\hline \multicolumn{2}{|l|}{ Mie-Grüneisen parameters } \\
\hline $\mathrm{S}_{1}$ & 2.56 \\
\hline $\mathrm{S}_{2}$ & -1.986 \\
\hline $\mathrm{S}_{3}$ & 0.2268 \\
\hline$\Gamma$ & 0.5 \\
\hline
\end{tabular}

Simulations are performed under three different loading conditions with varying degrees of stress triaxiality. The loading paths investigated included pure hydrostatic loading to probe the volumetric response in the absence of applied deviatoric stresses, a uniaxial strain loading path akin of loading conditions at the front of a propagating shock, and a divergent flow path which represents approximate conditions behind a shock front. In each case, initial conditions in the form of a spatially varying velocity field are applied, resulting in constant strain rates throughout the RVE. Different loading conditions are achieved by independently varying the strain rates in each of the three principal loading directions as shown in the table below:

\begin{tabular}{|l|l|l|l|}
\hline \multirow{2}{*}{$\begin{array}{l}\text { Loading } \\
\text { condition }\end{array}$} & \multicolumn{3}{|c|}{ Applied strain rate $\mathbf{s}^{-1}$ ) } \\
\cline { 2 - 4 } & $\dot{\varepsilon}_{x x}$ & \multicolumn{1}{|c|}{$\dot{\varepsilon}_{y y}$} & \multicolumn{1}{c|}{$\dot{\varepsilon}_{z z}$} \\
\hline Hydrostatic & $-0.5774 \dot{\varepsilon}$ & $-0.5774 \dot{\varepsilon}$ & $-0.5774 \dot{\varepsilon}$ \\
\hline 1D strain & $-\dot{\varepsilon}$ & 0 & 0 \\
\hline Divergent & $-0.96225 \dot{\varepsilon}$ & $0.19245 \dot{\varepsilon}$ & $0.19245 \dot{\varepsilon}$ \\
\hline In all loading cases above, $\dot{\varepsilon}=10^{5} \mathrm{~s}^{-1}$ \\
\hline
\end{tabular}

Simulation results are shown in Figure 21-Figure 24. Figure 21 depicts the volumetric response of dry and saturated sand in a pressure-density space for all three loading conditions. This figure shows a rather striking difference between the volumetric response of dry and saturated sand. For saturated sand, the volumetric response appears to be independent of stress triaxiality, with all three of the load paths investigated exhibiting nearly identical volumetric behavior. On the other hand, the response of dry sand appears to be path-dependent, exhibiting different responses under different loading conditions. In particular, our simulation results indicate that the pressure required to dynamically compact the RVE to a given density increases with decreasing stress triaxiality, a response feature commonly known as shear-enhanced compaction. The reasons why shear-enhanced compaction is present in the simulations of the compaction of dry sand, and not in the simulations of the compaction of wet sand are not yet fully understood, and they require further investigation. 


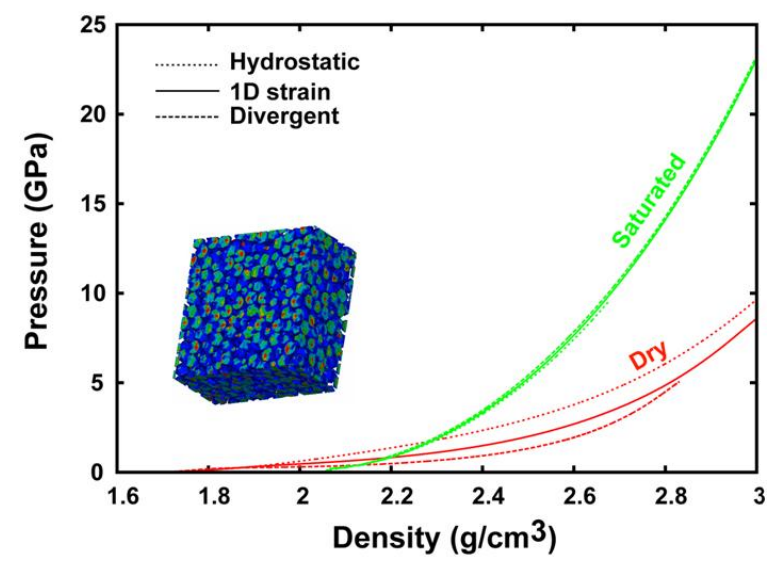

Figure 21: Volumetric response of dry and saturated granular silica $\left(\mathrm{SiO}_{2}\right)$ under hydrostatic, uniaxial strain, and spherically divergent loading conditions.

Figure 22 shows the pressure-dependent yield surface of $\mathrm{SiO}_{2}$ (solid sand), along with the computed response of dry and saturated sand under 1D strain and divergent loading conditions. For all the loading conditions investigated, the yield strength of sand is significantly lower than that of $\mathrm{SiO}_{2}$, with the strength of dry sand expectedly approaching the strength of the solid $\mathrm{SiO}_{2}$ at high dynamic pressure. Figure 3 also shows that when plotted in terms of total pressure, the strength of the saturated material is consistently lower than the strength of the dry material, irrespective of stress triaxiality or the magnitude of the applied pressure.

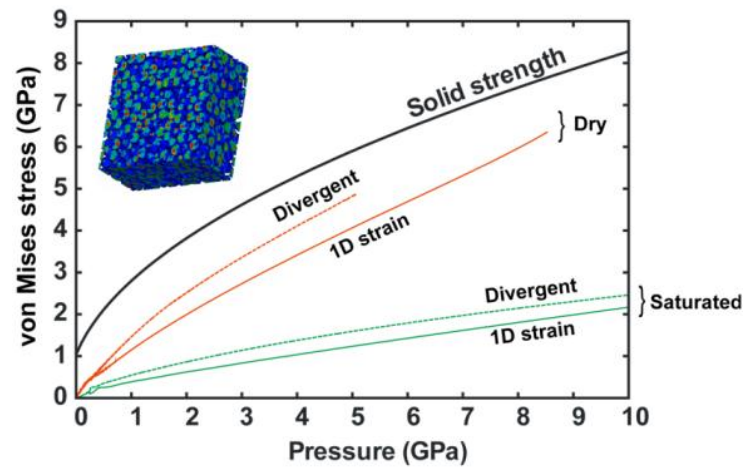

Figure 22: Dependence of yield strength on pressure under dry and saturated conditions.

The same sand strength data that was shown in Figure 22 are also shown in Figure 23. In addition, Figure 23 also shows the strength of saturated sand plotted as a function of effective stress. In the figure, the green curves are the same as the ones from Figure 22 . The blue curves are the same results, plotted in terms of effective stress. One can now compare the red, which represents the dry material response, to the blue, which represents saturated response, both plotted in terms of effective stress. If the effective stress model applies to this system, the blue curves would overlay the corresponding red curves. The two response curves actually do this at very low pressures, but they deviate considerably as the pressure increases. This indicates that at low pressure, where the effective stress model has been extensively 
validated, the model accurately represents the material behavior. However, as the pressures are increased, the material response deviates considerably from that implied in the effective stress model, and significant modification to that model will be required to capture the response features observed in the mesoscale simulations. Additionally, the results shown in Figure 23 indicate that saturated sand behaves stronger than the effective stress model indicates, and in terms of effective stress the saturated medium is stronger than the unsaturated medium. However, as was shown in Figure 22, in terms of applied total pressure, the strength of the dry medium is always higher than that of its saturated counterpart.

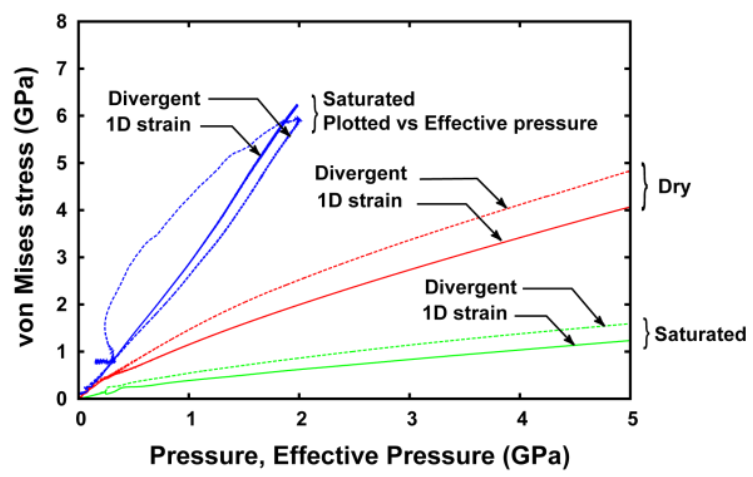

Figure 23: Yield surfaces for dry and saturated $\mathrm{Si}_{2}$ under different loading conditions plotted as a function of both applied and effective pressure.

Figure 24 depicts the relationship between effective pressure and total pressure for all the cases investigated in the present study. The linear behavior exhibited by dry sand is expected, confirming that in the absence of fluid, the effective pressure is identically equal to the applied pressure. However, the precipitous drop in effective pressure starting at an applied pressure level of about $40 \mathrm{GPa}$ is unexpected, and cannot be correlated to changes in the behavior of solid $\mathrm{SiO}_{2}$ at those pressure levels. This anomaly is not yet fully understood, and as such requires further investigation.

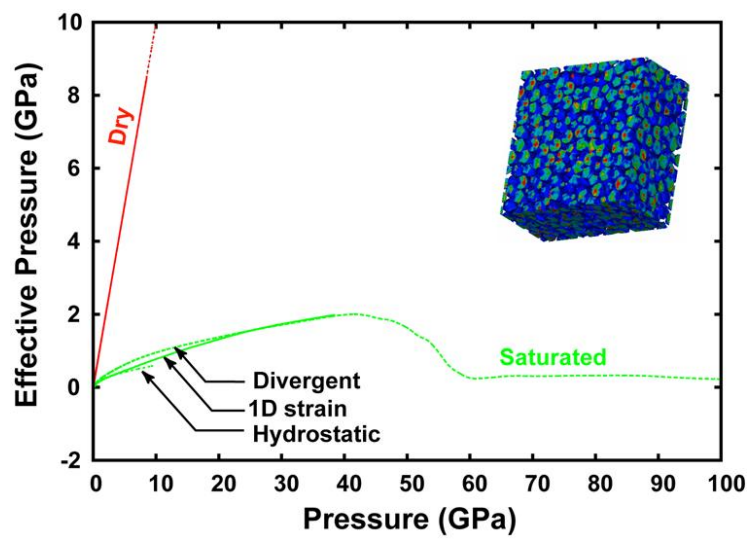

Figure 24: Dependence of effective pressure on applied pressure. 


\section{Upcoming Tasks}

\section{Finish Comparison with SHPB Tests}

Preliminary work towards direct numerical simulation of Split Hopkinson Pressure Bar (SHPB) experiments using a mesoscale representation of each individual grain is currently underway. The initial model has been constructed and is currently being evaluated. Figure 25 shows the SHPB experimental configuration from Kabir, Martin and Chen [8] and the current state of the simulations. In the simulations $\sim 2700$ particles are placed within a confining tube and surrounded by Al platens, which act as a pulse shaping device. The time-sequenced images from the simulations are images of a semitransparent apparatus with a cut-plane through its length. The pulse travels from left to right and prominent force chains emerge after the reflected pulse from the right Al platen compact the sample further. Future simulations must include properly scaled lengths of the incident and transmission bars and include an appropriate striker bar (or boundary condition) that will provide similar input pulses to the sample.
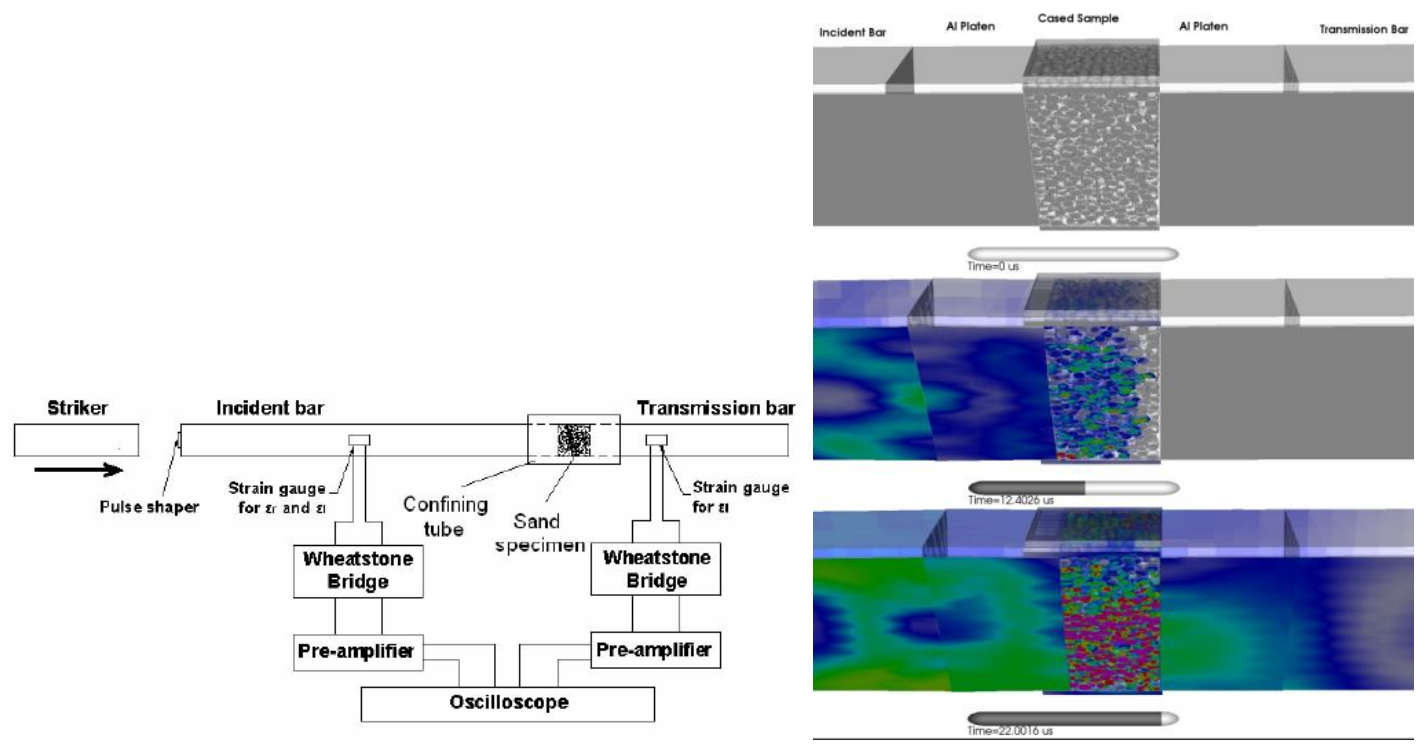

Figure 25: Modeling of Split Hopkinson Pressure Bar experiments for the configuration (from Figure 2 XXXX) and results of the finite element model.

\section{Implement Enhanced Sand Models into ALE3D}

The contact element library associated with the finite element modeling of granular material has been migrated into the ALE3D code base. A continuum scale model of granular material based on evolving moments of the size distribution is also being co-developed between this project leveraged with an internal effort to develop failure and post-failure models of concrete. The model is being informed by mesoscale simulations of concrete fracture and comminution for the internal effort, while a similar procedure using the mesoscale studies detailed here is being used to inform the evolving damage behavior of dynamically loaded sand.

\section{Model Scaled Penetrator Impact Tests}

This task is scheduled to start in the latter part of FY12 and will last through mid-FY14. 


\section{Simulate Full-Scale Penetration Experiments}

This task is scheduled to start in FY14-Q2 and will last through FY15.

\section{References}

[1] M. E. Backman and W. Goldsmith, "The mechanics of penetration of projectiles into targets," International Journal of Engineering Science, vol. 16, no. 1, pp. 1-99, 1978.

[2] J. L. Brown, T. J. Vogler, L. C. Chhabilidas, T. F. Thornhill, and W. D. Reinhart, "Shock response of dry sand," Albuquerque, NM, Aug. 2007.

[3] R. A. Graham, "SHOCK-WAVE COMPRESSION OF x-CUT QUARTZ AS DETERMINED BY ELECTRICAL RESPONSE MEASUREMENTS," Journal of Physics and Chemistry of Solids, vol. 35, pp. 355-372, 1974.

[4] J. J. Gallagher, Jr., "Fracturing of Quartz Grains," Tulsa, OK, 1976.

[5] W. A. Allen, E. B. Mayfield, and H. L. Morrison, "Dynamics of a Projectile Penetrating Sand," Journal of Applied Physics, vol. 28, no. 3, pp. 370-376, 1957.

[6] W. A. Allen, E. B. Mayfield, and H. L. Morrison, "Dynamics of a Projectile Penetrating Sand. Part II," Journal of Applied Physics, vol. 28, no. 11, pp. 1331-1335, 1957.

[7] W. L. Cooper and B. a. Breaux, "Grain fracture in rapid particulate media deformation and a particulate media research roadmap from the PMEE workshops," International Journal of Fracture, vol. 162, no. 1-2, pp. 137-150, Mar. 2010.

[8] M. E. Kabir, B. Song, B. E. Martin, and W. Chen, "Compressive Behavior of Fine Sand," 2010.

[9] J. L. Brown, T. J. Vogler, D. E. Grady, W. D. Reinhart, L. C. Chhabilidas, and T. F. Thornhill, "Dynamic compaction of sand," in Shock Compression of Condensed Matter, 2007, pp. 13631366.

[10] K. L. Johnson, Contact Mechanics. Cambridge, UK: Cambridge University Press, 1987, p. 452.

[11] T. Wichtmann and T. Triantafyllidis, "Influence of the Grain-Size Distribution Curve of Quartz Sand on the Small Strain Shear Modulus Gmax," Journal of Geotechnical and Geoenvironmental Engineering, vol. 135, no. 10, pp. 1404-1418, 2009.

[12] J. P. Morris and S. M. Johnson, Simulations of fracture and fragmentation of geologic materials using combined FEM/DEM/SPH analysis. MEI Conferences, 2007.

[13] K. T. Chau, X. X. Wei, R. H. C. Wong, and T. X. Yu, "Fragmentation of brittle spheres under static and dynamic compressions : experiments and analyses," Mechanics of Materials, vol. 32, pp. 543-554, 2000.

[14] S. Karner, "Subcritical compaction and yielding of granular quartz sand," Tectonophysics, vol. 377, no. 3-4, pp. 357-381, Dec. 2003.

[15] F. A. Chuhan, A. Kjeldstad, K. Bjorlykke, and K. Hoeg, "Porosity loss in sand by grain crushingexperimental evidence and relevance to reservoir quality," Marine and Petroleum Geology, vol. 19, pp. 39-53, 2002.

[16] J. P. Borg, R. Lewis, and B. Plunkett, "A comparison of two and three dimensional multiscale simulation as applied to porous heterogeneous materials," in Royal Society of London, 2010, p. 45.

[17] M. E. Kabir, B. Song, B. E. Martin, and W. Chen, "Compressive Behavior of Fine Sand," Albuquerque, NM, 2010.

[18] D. He, N. N. Ekere, and L. Cai, "Computer simulation of random packing of unequal particles," Physical Review E, vol. 60, no. 7098, 1999. 